\title{
Cambios en la estructura química del polietileno de alta densidad al experimentar múltiples reprocesamientos*
}

\author{
Juan Cárdenas-Giraldo* \\ Andrés Rojas-González ${ }^{* * *}$ \\ Beatriz Gálviz-García***
}

Recibido: 24/01/2018 - Aceptado: 20/06/2019

https://doi.org/10.22395/rium.v18n35a7

\begin{abstract}
Resumen
En este artículo, que presenta los resultados originales de una investigación financiada por Colciencias y la Facultad de Ingeniería y Arquitectura de la Universidad Nacional de Colombia, sede Manizales, se identifican los cambios en la estructura química del polietileno de alta densidad grado extrusión y grado inyección después de ser sometidos a cinco reprocesamientos en procesos de extrusión, inyección y extrusión/inyección. La identificación de los cambios se realiza a través de la comparación del espectro FTIR con los reportados en diferentes referencias bibliográficas. Los espectros FTIR fueron obtenidos mediante un espectrómetro ATR Platinum. La degradación térmica fue mayor en el proceso de extrusión/inyección, seguido por inyección, extrusión, extrusión a $80 \mathrm{rpm}$ y extrusión a $20 \mathrm{rpm}$. Se concluye que existe una baja degradación del polietileno de alta densidad después de ser reprocesado en cinco ciclos, se evidencia así la aparición del grupo carbonilo, grupos vinilo y grupo COC.
\end{abstract}

Palabras clave: degradación térmica; FTIR; reprocesamiento en inyección; reprocesamiento en extrusión; polietileno de alta densidad.

* Resultado de investigación n. 34958 financiada por Colciencias y la Facultad de Ingeniería y Arquitectura de la Universidad Nacional de Colombia, sede Manizales.

** Ingeniero químico e ingeniero industrial, especialista en Ingeniería Ambiental. Auxiliar de investigación del Grupo de Investigación Aprovechamiento de Residuos (GIAR), Universidad Nacional de Colombia, Manizales, Colombia. Correo electrónico: jumcardenasgi@unal.edu.co

*** Ingeniero químico, magíster en Ingeniería Química, doctor en Ingeniería Química. Profesor asociado, Universidad Nacional de Colombia, Manizales, Colombia. Correo electrónico: anfrojasgo@unal.edu.co. Orcid: http://orcid. org/0000-0002-6905-0802

**** Ingeniera física, magíster en Ciencias Físicas, Universidad Nacional de Colombia, Manizales, Colombia. Correo electrónico: bcgalvizg@unal.edu.co 


\title{
Changes in the Chemical Structure of High-Density Polyethylene when Undergoing Multiple Reprocessing
}

\begin{abstract}
In this paper, which presents the original results of a research funded by Colciencias and Facultad de Ingeniería y Arquitectura from the Universidad Nacional de Colombia, headquarters Manizales, publishes the changes of high-density polyethylene extrusion and injection grade identified after being subjected to five reprocessing processes in extrusion, injection and extrusion/ injection. The identification of the changes is made through the comparison of the FTIR spectrum with those reported in different bibliographical references. The spectra were obtained by means of a Platinum ATR spectrometer. The thermal degradation was greater in the extrusion/injection process, followed by injection, extrusion at $80 \mathrm{rpm}$ and extrusion at $20 \mathrm{rpm}$. It is concluded that there is a low degradation of high-density polyethylene after being reprocessed in five cycles, evidencing the appearance of the carbonyl group, vinyl groups and COC group.
\end{abstract}

Keywords: thermal degradation; FTIR; injection reprocessing; extrusion reprocessing; high-density polyethylene.

\section{Mudanças na estrutura química do polietileno de alta densidade ao experimentar múltiplos reprocessamentos}

\begin{abstract}
Resumo
Neste artigo, que apresenta os resultados originais de uma pesquisa financiada por Colciencias e pela Faculdade de Engenharia e Arquitetura da Universidad Nacional de Colombia, sede Manizales, são identificadas as mudanças na estrutura química do polietileno de alta densidade grau extrusão e grau injeção depois de serem submetidos a cinco reprocessamentos em processos de extrusão, injeção e extrusão/injeção. A identificação das mudanças é realizada por meio da comparação do espectro FTIR com o que foi relatado em diferentes referências bibliográficas. Os espectros FTIR foram obtidos por meio de um espectrômetro ATR Platinum. A degradação térmica foi maior no processo de extrusão/injeção, seguido por injeção, extrusão, extrusão a 80 rpm e extrusão a $20 \mathrm{rpm}$. Conclui-se que existe uma baixa degradação do polietileno de alta densidade depois de ser reprocessado em cinco ciclos, assim fica evidente o surgimento do grupo carbonila, grupos vinilo e grupo COC.
\end{abstract}

Palavras-chave: degradação térmica; FTIR; reprocessamento em injeção; reprocessamento em extrusão; polietileno de alta densidade. 


\section{INTRODUCCIÓN}

En los últimos 50 años la producción mundial de plásticos ha incrementado significativamente. Se estima que los residuos generados por la disposición de estos materiales se encuentran cerca a 150 millones de toneladas al año [1]. Debido a la baja relación peso/ volumen, la alta producción y la difícil degradabilidad de la mayoría de los polímeros, la disposición de dichos residuos en rellenos sanitarios no es una buena opción [2].

Los métodos industriales más usados para el procesamiento y reprocesamiento de polímeros son la extrusión y la inyección $[1,3]$. El reprocesamiento consiste en la reincorporación de material ya procesado al ciclo normal de inyección o extrusión que reduce así la cantidad de residuos generados [4]. Durante el proceso de inyección y extrusión es posible que el material sufra cambios químicos y físicos por efectos de la temperatura, el estrés mecánico y la oxidación. Algunos autores establecen el límite de reprocesamientos para un polímero virgen en dos o tres, debido a variaciones en sus propiedades mecánicas tales como la resistencia, que se ve reducida por la degradación térmica, mecánica u oxidativa $[1,5]$. Los métodos que se han usado para evaluar dichos cambios incluyen: calorimetría diferencial de barrido (DSC) [1, 5-12]; análisis termogravimétrico (TGA) [1-2, 5, 7-8, 10-13]; microscopía electrónica de barrido (SEM) [5]; cromatografía por permeación de gel (GPC) [7-8, 11-12]; espectroscopía de resonancia magnética nuclear (NMR) [5]; reología [1, 5, 7]; cromatografía de gases (GC) [2, 14]; espectroscopía de masas (MS) [2]; índice de fluidez (MFI) [4-5, 7-8, 11]; evaluación de propiedades mecánicas tales como el módulo de Young; resistencia al impacto; resistencia máxima $[1,5,7-8,11-12,14]$ y espectroscopia por transformada de Fourier (FTIR) [7-8, 10-13, 15].

La técnica FTIR, junto con alguno de los métodos anteriores o en forma separada, se ha utilizado ampliamente en la caracterización e identificación química de polímeros, debido a su facilidad de uso, rapidez y confiabilidad [1-2, 11-13, 15]. Existen diversas técnicas de muestreo en el análisis por FTIR, como: técnicas por transmisión, técnicas por reflectancia y espectroscopía fotoacústica. Las técnicas por reflectancia comprenden la reflectancia difusiva (Drifts), reflectancia especular y reflectancia total atenuada (ATR) [16], siendo esta última de especial importancia, ya que no requiere prácticamente preparación de la muestra y permite cuantificar el índice de carbonilo en la oxidación de polioleofinas tales como el polietileno de alta densidad (HDPE) [9, 17].

El polietileno de alta densidad (HDPE) es uno de los polímeros más consumidos a nivel mundial, esto se debe a su uso principal como materia prima en diferentes productos plásticos y al bajo costo comparado con sus buenas propiedades mecánicas y químicas $[1,11]$. A pesar de esto, existen pocos estudios relacionados con la variación de la estructura química del HDPE después de ser reprocesado en varios ciclos [7-8]. 
Estudios anteriores han permitido determinar la variación en propiedades físicas y cambios estructurales del HDPE como consecuencia de la degradación térmica, mecánica y oxidativa $[2,3-5,7,10-11]$. Se ha establecido que este polímero presenta principalmente degradación por rompimiento de cadena debido a efectos de la temperatura y por ramificación y/o entrecruzamiento debido a estrés mecánico a bajas temperaturas $[6,18]$. Otros autores establecen que el mecanismo principal de degradación en las primeras 30 extrusiones se debe a una ramificación de la cadena y en las sucesivas extrusiones, a rompimientos de esta. También sugieren que a partir de 60 extrusiones pueden aparecer entrecruzamientos de la cadena [19].

El HDPE se ha analizado a través de FTIR, esto con el fin de caracterizarlo y diferenciarlo de las demás polioleofinas, tales como el polietileno de baja densidad (LDPE) y el polietileno lineal de baja densidad (LLDPE) [15]. También se ha utilizado la técnica de FTIR para la identificación y análisis de cambios sufridos por degradación térmica, oxidativa y/o mecánica. En estos análisis se ha identificado la presencia de grupos terminales vinilideno, compuestos del grupo carbonilo y vinilos que en diversas condiciones son producto normal del procesamiento industrial $[7,11,20]$.

El objetivo de este trabajo es identificar a través de FTIR las posibles variaciones en la estructura química del polietileno de alta densidad, grado inyección y grado extrusión después de reprocesarlos en cinco ciclos. Estos polímeros se sometieron a los procesos de extrusión, inyección y extrusión/inyección. Como parámetros de análisis se considera: i) número de reprocesamientos (cinco ciclos); ii) tipo de proceso (extrusión, inyección y extrusión/inyección); y iii) la velocidad del husillo en el proceso de extrusión ( 20 y $80 \mathrm{rpm})$.

\section{MATERIALES Y MÉTODOS}

\subsection{Caracterización del polímero virgen y reprocesado}

El polietileno de alta densidad grado inyección y grado extrusión fue suministrado por la empresa IDES Prospector - USA en forma de pellets. La tabla 1 presenta algunas propiedades del polímero según los datos suministrados por el fabricante. La caracterización y evaluación de los cambios en la estructura química del polímero virgen y de las muestras reprocesadas se llevaron a cabo mediante espectroscopia infrarroja por transformada de Fourier (FTIR) en un espectrómetro marca Bruker, con accesorio Alpha y ATR Platinum Diamond. El barrido del espectro se realizó en un rango de número de onda de 400 a $4.000 \mathrm{~cm}^{-1}$, con una incertidumbre o desviación de $0,22 \mathrm{~cm}^{-1}$, una resolución de $0,4 \mathrm{~cm}^{-1}$ y un escaneo de 32 . 
Tabla 1. Propiedades del polímero según el fabricante

\begin{tabular}{lcc}
\hline \multicolumn{1}{c}{ Parámetro } & HDPE-grado inyección & HDPE-grado extrusión \\
\hline Índice de fluidez $190^{\circ} \mathrm{C} / 2,16 \mathrm{~kg}, \mathrm{~g} / 10 \mathrm{~min}$ & 37 & 0,38 \\
\hline Temperatura de transición vítrea, ${ }^{\circ} \mathrm{C}$ & -113 & -111 \\
\hline Temperatura de fusión o de ablandamiento, ${ }^{\circ} \mathrm{C}$ & 129 & 131 \\
\hline Temperatura de cristalización, ${ }^{\circ} \mathrm{C}$ & 113 & 118 \\
\hline Tipo de polímero & Semicristalino & Semicristalino \\
\hline Grado de cristalinidad, $\%$ & 59,35 & 70,34 \\
\hline
\end{tabular}

Fuente: elaboración propia

\subsection{Procesamiento del polímero}

Las muestras de polietileno de alta densidad grado inyección (HDPE-GI) y grado extrusión (HDPE-GE) fueron reprocesadas cinco veces en cada uno de los siguientes tres procesos: i) extrusión, ii) inyección, y iii) extrusión/inyección. Un reprocesamiento o ciclo de reprocesamiento equivale a la secuencia de cada una de las etapas siguientes: secado inicial según las condiciones del fabricante; extrusión, inyección o extrusión/ inyección; enfriamiento mediante baño de agua; reducción del tamaño o peletizado a través de molino de cuchillas; y secado final en estufa según condiciones del fabricante. El proceso de extrusión se llevó a cabo en una extrusora monohusillo marca Extrudex modelo ED-N 45-30D, con un diámetro del husillo de $45 \mathrm{~mm}, 8$ zonas de calentamiento, una relación L/D de 30 y una restricción en la zona de dosificación entre 0 y 56 . Este proceso se realizó para los dos polímeros (HDPE-GI y HDPE-GI). Las condiciones de reprocesamiento para la extrusión se presentan en la tabla 2.

Tabla 2. Condiciones de operación en la extrusión para el reprocesamiento del HDPE, grado inyección y grado extrusión

\begin{tabular}{|l|c|c|}
\hline \multicolumn{1}{|c|}{ Parámetro } & HDPE-grado inyección & HDPE-grado extrusión \\
\hline Temperatura cilindro $1,{ }^{\circ} \mathrm{C}$ & 180 & 180 \\
\hline Temperatura cilindro $2,{ }^{\circ} \mathrm{C}$ & 190 & 190 \\
\hline Temperatura cilindro $3,{ }^{\circ} \mathrm{C}$ & 200 & 200 \\
\hline Temperatura cilindro $4,{ }^{\circ} \mathrm{C}$ & 210 & 210 \\
\hline Temperatura brida, ${ }^{\circ} \mathrm{C}$ & 220 & 220 \\
\hline Temperatura homogenización $1,{ }^{\circ} \mathrm{C}$ & 220 & 220 \\
\hline Temperatura homogenización $2,{ }^{\circ} \mathrm{C}$ & 220 & 220 \\
\hline Temperatura alimentación, ${ }^{\circ} \mathrm{C}$ & 28 & 28 \\
\hline Velocidad del tornillo, rpm & 30 & $20 \mathrm{y} 80$ \\
\hline Restricción & 10 & 10 \\
\hline
\end{tabular}

Fuente: elaboración propia 
El HDPE-GI fue reprocesado adicionalmente por inyección y a través de un proceso de extrusión/inyección. No fue posible realizar este mismo procedimiento para el HDPE-GE, dado que este polímero presentaba problemas al momento de realizar la operación, debido a su bajo índice de fluidez $(0,38 \mathrm{~g} / 10 \mathrm{~min})$. El proceso de inyección se llevó a cabo en una inyectora monohusillo marca Boy XS, con un diámetro del husillo de $12 \mathrm{~mm}, 4$ zonas de calentamiento, una relación L/D de 19,6, una velocidad máxima de inyección de $24 \mathrm{~cm}^{3} / \mathrm{s}$ y una presión máxima de 3130 bar (45,4 psi). La tabla 3 presenta las condiciones de reprocesamiento para la operación de inyección.

Tabla 3. Condiciones de operación en la inyección

para el reprocesamiento del HDPE grado inyección

\begin{tabular}{lc}
\hline \multicolumn{1}{c}{ Parámetro } & HDPE-grado inyección \\
\hline Temperatura del molde, ${ }^{\circ} \mathrm{C}$ & 12 \\
\hline Temperatura de la boquilla, ${ }^{\circ} \mathrm{C}$ & 180 \\
\hline Temperatura plastificación $1,{ }^{\circ} \mathrm{C}$ & 220 \\
\hline Temperatura plastificación $2,{ }^{\circ} \mathrm{C}$ & 200 \\
\hline Tiempo de inyección, s & 6 \\
\hline Tiempo de enfriamiento, s & 28 \\
\hline Velocidad del tornillo, rpm & 75 \\
\hline
\end{tabular}

Fuente: elaboración propia

\subsection{Análisis de los espectros FTIR}

Los 28 espectros obtenidos por FTIR fueron comparados uno a uno con los picos representativos, estos corresponden a números de onda característicos para el HDPE o a posibles productos de la degradación térmica, mecánica u oxidativa. Los picos representativos para el polietileno de alta densidad se presentan en la tabla 4 . La comparación de los espectros con la tabla 4 permite identificar fácilmente los picos y brinda un marco de análisis a través de las referencias bibliográficas para los espectros FTIR obtenidos.

\section{RESULTADOS Y DISCUSIÓN}

La figura 1 presenta los espectros FTIR para los polímeros grado inyección (HDPEGI) y grado extrusión (HDPE-GE) sin reprocesar (polímeros vírgenes). Los espectros de la figura 1; al igual que para los espectros del HDPE-GI sin reprocesar (HDPE0), extruido 5 veces (HDPE-E5), inyectado 5 veces (HDPE-I5) y extruido/inyectado en 5 ciclos (HDPE-EI5) de la figura 2; y para el HDPE-GE sin reprocesar (HDPE0), 
extruido 5 veces a $20 \mathrm{rpm}$ (HDPE-E20-5) y extruido 5 veces a $80 \mathrm{rpm}$ (HDPE-E80-5) de la figura 3 se caracterizan por presentar 4 picos correspondientes al grupo funcional alifático $\mathrm{CH}_{2}-\mathrm{CH}_{2}$, que confirman que se está analizando el polímero HDPE. Dos de estos picos son de alta intensidad, localizados entre 2.980 y $2.750 \mathrm{~cm}^{-1}$, y dos picos de baja intensidad, uno de ellos entre 1.480 y $1.420 \mathrm{~cm}^{-1}$, y el otro entre 740 y $700 \mathrm{~cm}^{-1}$.

Tabla 4. Números de onda representativos en FTIR para el HDPE virgen o degradado

\begin{tabular}{|c|c|c|}
\hline Número de onda $\left(\mathrm{cm}^{-1}\right)$ & Descripción & Fuente \\
\hline $720-730$ & Vibraciones de flexión C-H & {$[12,15]$} \\
\hline 874,888 & Grupos terminales vinilideno $\left(\mathrm{R}_{1} \mathrm{R}_{2} \mathrm{C}=\mathrm{CH}_{2}\right)$ & {$[7,11,20]$} \\
\hline 907, 991 & Grupos vinilo $\left(-\mathrm{CH}=\mathrm{CH}_{2}\right)$ & {$[7,11,20]$} \\
\hline 964 & Radicales alquil secundarios (- $\mathrm{CH}=\mathrm{CH}-)$ & {$[7,20]$} \\
\hline 1048,1050 & Grupo COC & {$[6,12]$} \\
\hline 1176 & Deformación de ondulación & [15] \\
\hline 1306 & Deformación de torsión & {$[15]$} \\
\hline 1351 & Banda que diferencia al HDPE del LDPE & [15] \\
\hline 1366,1351 & Deformación de ondulación & {$[15]$} \\
\hline 1377 & Deformación simétrica del $\mathrm{CH}_{3}$ & {$[15,20]$} \\
\hline $1400-1550$ & Grupo metileno & {$[5]$} \\
\hline 1411 & Formación de cetonas internas & {$[5]$} \\
\hline 1463,1473 & Deformación de flexión & {$[15]$} \\
\hline 1490 & Vibración de flexión de C-H & {$[12]$} \\
\hline $1650-1800$ & Grupo carbonilo & {$[5,7,11]$} \\
\hline 1700 & Ácidos carboxílicos & {$[5]$} \\
\hline 1716 & Cetonas & {$[5]$} \\
\hline 1741 & Aldehídos y cetonas & {$[5]$} \\
\hline 1783 & Lactonas & {$[5]$} \\
\hline 2851 & Extensión simétrica de $\mathrm{CH}_{2}$ & {$[12,15]$} \\
\hline 2919 & Extensión asimétrica de $\mathrm{CH}_{2}$ & {$[12,15]$} \\
\hline
\end{tabular}

Fuente: elaboración propia

En los resultados obtenidos de FTIR para los polímeros sin reprocesar según la figura 1 y la comparación detallada de picos (figura 4), se encuentra que no existe diferencia significativa en la estructura química del HDPE-GI y el HDPE-GE. Esto 
se debe a que se tiene un mismo polímero con un grado de cristalinidad diferente, la cual genera una diferencia importante en el índice de fluidez entre ellos, pero no en la composición base de los grupos funcionales, como el grupo funcional alifático $\mathrm{CH}_{2}-\mathrm{CH}_{2}$-, típicos del HDPE.

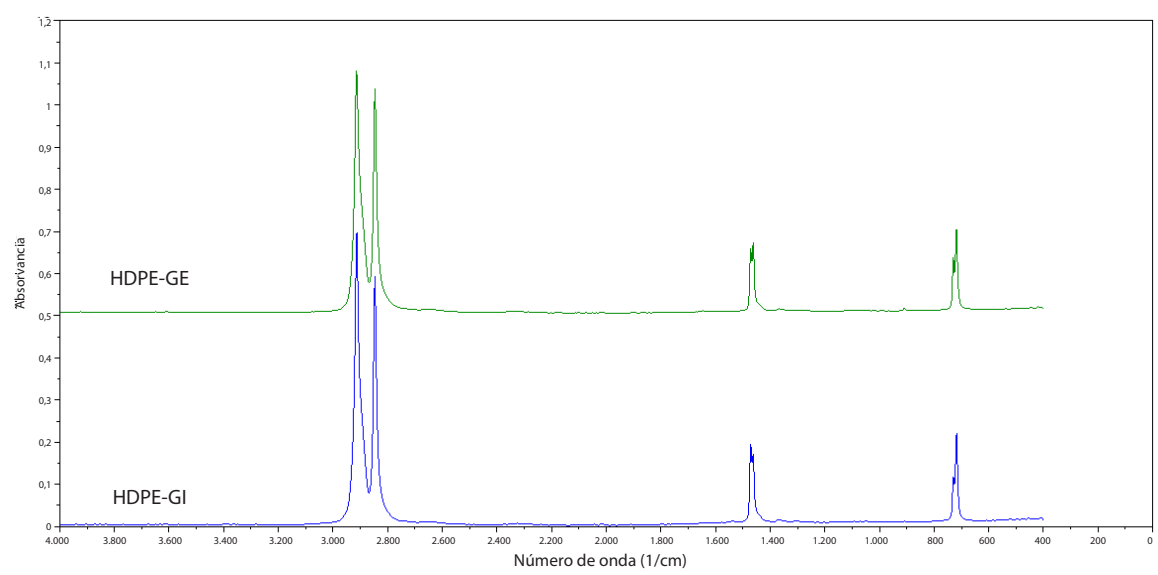

Figura 1. Espectros FTIR para los polímeros grado inyección (HDPE-GI) y grado extrusión (HDPE-GE) sin reprocesar Fuente: elaboración propia

Los resultados obtenidos del análisis FTIR para el reprocesamiento del HDPE grado inyección reprocesado 5 veces en inyección, extrusión y extrusión/inyección se presentan en la figura 2 .

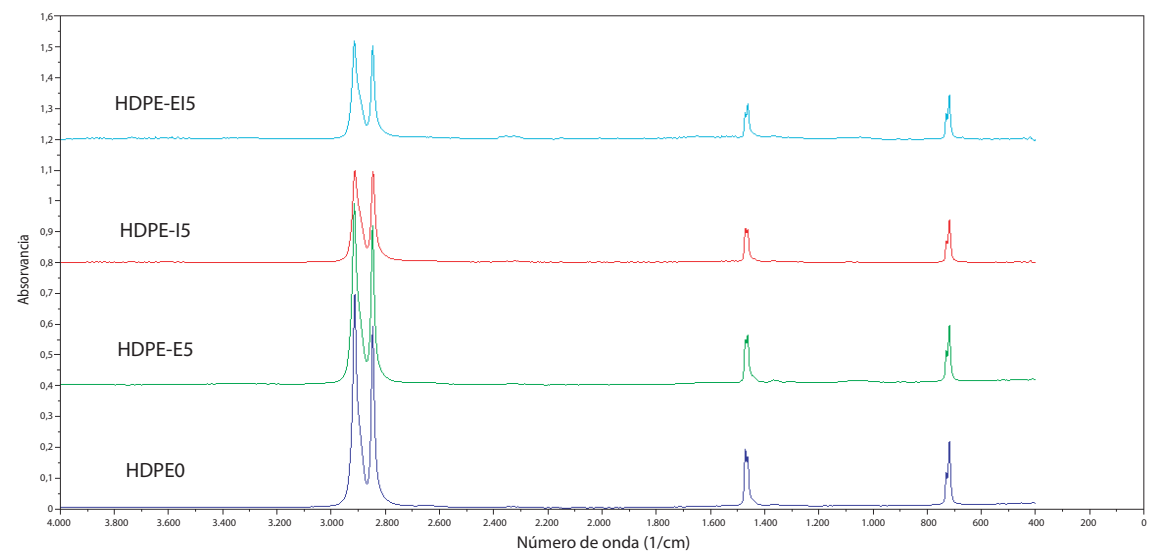

Figura 2. Espectros FTIR para el HDPE-GI sin reprocesar (HDPE0), extruido 5 veces (HDPE-E5), inyectado 5 veces (HDPE-15) y extruido/inyectado en 5 ciclos (HDPE-El5). 
En esta figura se observa que la intensidad de la absorbancia es similar entre el polímero sin reprocesar y el reprocesado por extrusión; mientras que la intensidad de la absorbancia es menor en los polímeros reprocesados por inyección y extrusión/inyección. Esto indica que la concentración del grupo funcional se reduce con el reprocesamiento, y que el proceso de inyección, solo o acompañado con el proceso de extrusión, afecta considerablemente la concentración de este grupo funcional $[5,11]$.

La disminución de la absorbancia en las muestras reprocesadas concuerda con lo reportado en la literatura [6]. La similitud en la intensidad de la absorbancia entre el polímero sin reprocesar y el reprocesado por extrusión indican que no hay diferencias significativas, por lo tanto, se puede establecer que el HDPE-GI no se ve considerablemente afectado (degradación despreciable) por los cinco ciclos de reprocesamiento en el proceso de inyección. Esto coincide con lo presentado en la literatura para las mismas muestras, en la que se estableció la degradación térmica por medio del índice de degradación en proceso, que está en función del índice de fluidez del polímero [4] y por análisis de imagen dada por el cambio de color [3].

El HDPE grado extrusión se reprocesó a una velocidad del giro del husillo de 20 rpm y $80 \mathrm{rpm}$ por medio del proceso de extrusión. Los resultados del análisis FTIR para estas dos velocidades de giro se presentan en la figura 3. En esta figura se observa que la velocidad de giro del husillo en el proceso de extrusión no afecta la estructura química del HDPE.

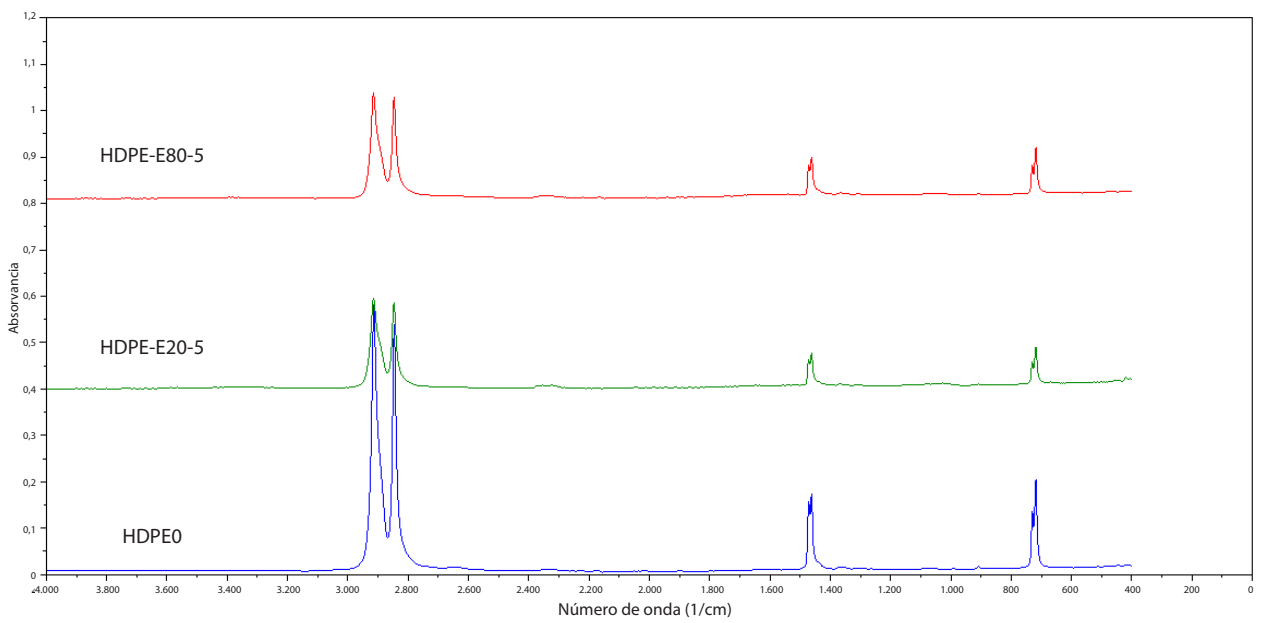

Figura 3. Espectros FTIR para el HDPE-GE sin reprocesar (HDPE0), extruido 5 veces a $20 \mathrm{rpm}$ (HDPE-E20-5) y extruido 5 veces a 80 rpm (HDPE-E80-5) 
La comparación de los espectros presentados en las figuras 2 y 3 no permite obtener información clara debido a la similitud entre ellos, lo que indica que no existe una degradación térmica, mecánica u oxidativa considerable. Sin embargo, comparando pico a pico en los espectros se puede obtener información adicional como la presencia de grupos que no son fácilmente identificables a través de las figuras anteriores. Para ello se construye la tabla 5, en la cual se presenta un resumen de los picos presentes en cada uno de los polímeros. La letra X identifica la existencia de un pico a una determinada longitud de onda.

Tabla 5. Matriz de picos presentes en todas las muestras analizadas mediante FTIR

\begin{tabular}{|c|c|c|c|c|c|c|c|c|c|c|c|c|c|c|c|c|c|c|c|c|c|c|c|c|c|c|c|}
\hline \multirow{2}{*}{$\begin{array}{c}\text { Número de } \\
\text { onda }\end{array}$} & \multicolumn{16}{|c|}{ Grado inyección } & \multicolumn{11}{|c|}{ Grado extrusión } \\
\hline & 0 & E1 & $E 2$ & E3 & E4 & E5 & II & $I 2$ & $I 3$ & I4 & $I 5$ & EII & EI2 & EI3 & EI4 & EI5 & 0 & E20-1 & E20-2 & E20-3 & E20-4 & E20-5 & $E 80-1$ & E80-2 & $E 80-$ & $E 80-4$ & E80-5 \\
\hline 717,86 & $\mathrm{X}$ & $X$ & $\mathrm{X}$ & $X$ & $\mathrm{X}$ & $\mathrm{X}$ & $X$ & $\mathrm{X}$ & $\mathrm{X}$ & $\mathrm{X}$ & $X$ & $X$ & $\mathrm{X}$ & $\mathrm{X}$ & $X$ & $\mathrm{X}$ & $\mathrm{X}$ & $\mathrm{X}$ & $X$ & $X$ & $\mathrm{X}$ & $\mathrm{X}$ & $\mathrm{X}$ & $\mathrm{X}$ & $\mathrm{X}$ & $\mathrm{X}$ & $\mathrm{X}$ \\
\hline 730,10 & $\mathrm{X}$ & $\mathrm{X}$ & $\mathrm{X}$ & $X$ & $\mathrm{X}$ & $\mathrm{X}$ & $\mathrm{X}$ & $\mathrm{X}$ & $X$ & $\mathrm{X}$ & $X$ & $X$ & $X$ & $\mathrm{X}$ & $X$ & $\mathrm{X}$ & $\mathrm{X}$ & $\mathrm{X}$ & $X$ & $X$ & $\mathrm{X}$ & $\mathrm{X}$ & $X$ & $X$ & $\mathrm{X}$ & $\mathrm{X}$ & $\mathrm{X}$ \\
\hline 907,53 & $\mathrm{X}$ & $X$ & & & & & & & & & & & & & & & $\mathrm{X}$ & $\mathrm{X}$ & $\mathrm{X}$ & $X$ & $\mathrm{X}$ & $\mathrm{X}$ & $X$ & $\mathrm{X}$ & $X$ & $\mathrm{X}$ & $X$ \\
\hline 1048,25 & $\mathrm{X}$ & $\mathrm{X}$ & $X$ & $\mathrm{X}$ & $\mathrm{X}$ & $X$ & $\mathrm{X}$ & $\mathrm{X}$ & $\mathrm{X}$ & $X$ & $X$ & $X$ & $X$ & $\mathrm{X}$ & $X$ & $\mathrm{X}$ & & & & & & & & $\mathrm{X}$ & $\mathrm{X}$ & $\mathrm{X}$ & $X$ \\
\hline 1303,17 & $\mathrm{X}$ & $X$ & $X$ & $X$ & $X$ & $X$ & & & & & & & & & & & & & & & & & & & & & \\
\hline 1366,39 & $X$ & $X$ & $X$ & $X$ & $X$ & $X$ & $X$ & $\mathrm{X}$ & $X$ & $X$ & $X$ & $X$ & $X$ & $X$ & $X$ & $X$ & $X$ & $X$ & $X$ & $X$ & $\mathrm{X}$ & $X$ & $X$ & $X$ & $X$ & $X$ & $X$ \\
\hline 1462,24 & $X$ & $X$ & $X$ & $\mathrm{X}$ & $X$ & $X$ & $X$ & $X$ & $X$ & $X$ & $X$ & $X$ & $X$ & $X$ & $X$ & $X$ & $X$ & $X$ & $X$ & $X$ & $X$ & $X$ & $X$ & $X$ & $X$ & $X$ & $X$ \\
\hline 1470,40 & X & $X$ & $X$ & $X$ & $X$ & $X$ & $X$ & $X$ & $X$ & $X$ & $X$ & $X$ & $X$ & $X$ & $X$ & $X$ & $X$ & $X$ & $X$ & $X$ & $X$ & $X$ & $X$ & $\mathrm{X}$ & $X$ & $X$ & $X$ \\
\hline 1646,58 & & & & & & & $X$ & $X$ & $X$ & $X$ & X & $X$ & $X$ & $X$ & $X$ & $X$ & & & & & & $X$ & $X$ & $X$ & $X$ & $X$ & $\mathrm{X}$ \\
\hline 1682,59 & & & & & & & & & & & & & & & & & & & & & & X & & & & & \\
\hline 2846,99 & $X$ & $X$ & $X$ & $X$ & $X$ & $X$ & $X$ & $X$ & $X$ & $X$ & $X$ & $X$ & $X$ & $X$ & $X$ & $X$ & $X$ & $X$ & $X$ & $X$ & $X$ & $X$ & $X$ & $X$ & $X$ & $X$ & $\mathrm{X}$ \\
\hline 2914,29 & $X$ & $X$ & $X$ & $\mathrm{X}$ & X & $\mathrm{X}$ & $X$ & $X$ & $X$ & $X$ & $X$ & $X$ & $X$ & $X$ & $X$ & $X$ & $X$ & $X$ & $X$ & $X$ & $X$ & $X$ & $X$ & $X$ & $X$ & $X$ & $X$ \\
\hline
\end{tabular}

Diversos autores reportan un pico a 874 y $888 \mathrm{~cm}^{-1}$ correspondiente al grupo vinilideno que se presenta normalmente en la oxidación del polietileno de alta densidad [7, 11, 20]; cómo se puede ver en la tabla 5, este pico no está presente. El grupo vinilo que corresponde a un número de onda de $907 \mathrm{~cm}^{-1}$ solo aparece en las muestras del polímero virgen y el primer reprocesamiento en extrusión para el polímero de grado inyección, esto puede ser consecuencia de una reacción del grupo vinilo que se convierte a COC o a un grupo carbonilo. Sin embargo, para el polímero de grado extrusión este grupo está presente en todas las muestras. El grupo vinilo ha sido reportado por diversos autores y se puede presentar en el polímero virgen debido al mismo procesamiento durante la producción industrial. Este grupo representa una degradación leve caracterizada por el enlace doble que puede facilitar la oxidación del polímero $[7,11,20]$. 
A un número de onda de $964 \mathrm{~cm}^{-1}$ se ha reportado el grupo trans vinileno o radicales alquil secundarios en la degradación del polietileno de alta densidad [11, 20]. Como se puede observar en la tabla 5 , este grupo no se encuentra presente en ninguna de las muestras.

El grupo COC correspondiente al número de onda $1.048 \mathrm{~cm}^{-1}$ se identificó en todas las muestras del HDPE grado inyección, mientras que en el HDPE grado extrusión solo aparece después del segundo reprocesamiento a $80 \mathrm{rpm}$. La presencia de este grupo indica que se da una oxidación del polímero [12]. Este comportamiento tambien se reporta en trabajos similares al presente documento [6].

El número de onda del pico correspondiente a la deformación por torsión en el polietileno de alta densidad solo se presentó en el polímero grado inyección durante la extrusión. Se desconoce la razón de la ausencia de este pico en las otras muestras. Este pico no indica una degradación y suele estar presente en el polietileno de alta densidad [15].

Los números de onda de 1.646 y $1.682 \mathrm{~cm}^{-1}$ presentados en la tabla 5 se encuentran en la región del grupo carbonilo [21]. Estos picos solo se presentan para el polímero grado inyección en los procesos de inyección y extrusión/inyección y en el polímero grado extrusión en el quinto reprocesamiento a $20 \mathrm{rpm}$ y en todos los reprocesamientos a $80 \mathrm{rpm}$. El grupo carbonilo juega un papel importante en la identificación de la degradación y envejecimiento del polímero, esto puede ser cuantificado a través del índice de carbonilo. Su presencia puede indicar un mecanismo de degradación a través del rompimiento de cadena. Este grupo se ha reportado en diversos estudios sobre la degradación del polietileno de alta densidad [5-6, 11, 22].

En ninguna de las muestras se presentó un indicativo más de degradación u oxidación, ya que no están presentes en bandas correspondientes a cetonas, lactonas, ácidos carboxílicos, aldehídos o ésteres que normalmente se presentan en el polietileno de alta densidad parcialmente oxidado [5].

La velocidad del tornillo juega un papel significativo en la aparición del grupo COC y el grupo carbonilo, dado que el polímero sin reprocesar no presentaba dichos grupos, mientras que a 80 rpm se evidenció la aparición de estos grupos. Lo asnterior permite concluir que el efecto combinado de la temperatura y el estrés mecánico al que se encuentra sometido el polímero a $80 \mathrm{rpm}$ influyen en la aparición de grupos COC y carbonilo. La ausencia del grupo COC y la presencia tardía del grupo carbonilo a 20 rpm confirma el efecto combinado de la temperatura y el estrés mecánico sobre el polímero reprocesado. 
Con respecto al tipo de reprocesamiento es evidente que la mayor degradación se presenta en el proceso combinado de extrusión/inyección, seguido por el proceso de inyección y finalizando con el proceso de extrusión. Resultados similares se han reportado en [3]. El efecto del grado de polímero en el proceso no se pudo evaluar debido a la dificultad presentada durante el proceso de inyección del polietileno grado extrusión.

El número de reprocesamientos influye en el espectro FTIR del polímero y favorece la aparición o desaparición de grupos respecto al polímero sin reprocesar. Este cambio se puede ver en el primero, segundo o quinto reprocesamiento, según los resultados obtenidos para el HDPE.

Los resultados presentados con respecto a la baja degradación del polietileno de alta densidad después de ser reprocesado son consecuentes con la literatura, pues se ha reportado degradación térmica para el HDPE a partir de $290^{\circ} \mathrm{C}[2,7]$ y descomposición a partir de $450^{\circ} \mathrm{C}[7,12]$. Estos valores son menores a la temperatura de reprocesamiento, tanto para la inyección como para la extrusión. En [8] se reportó baja degradación del polietileno de alta densidad incluso para 50 reprocesamientos y en [6] -un estudio similar para 5 reprocesamientos- se obtuvieron resultados similares en cuanto a la baja degradación del polietileno de alta densidad. También se ha reportado poco cambio en el espectro FTIR del polímero original con respecto al reciclado mediante el proceso de disolución/precipitación [14]. El proceso de reciclado mediante disolución/precipitación puede generar mayor degradación que el simple reprocesamiento, sin embargo, incluso para el reciclado no se evidenció una degradación considerable.

Otros autores han reportado degradación y oxidación significativa del polietileno de alta densidad medida a través de FTIR, pero para el polímero reciclado post consumo y expuesto al medio ambiente [11]. En este trabajo no se presentó degradación significativa del HDPE debido a su naturaleza de polímero post industrial pero no post consumo, pues este no se había utilizado en productos ni había cumplido su ciclo de vida convencional.

El análisis de la degradación de polímeros o del cambio en la estructura química es un tema complejo debido a la infinidad de variables involucradas en su procesamiento, obtención y tratamiento. Por lo tanto, su predicción es muy difícil [8, 23].

\section{CONCLUSIONES}

Se establece que no existe una degradación significativa de los dos tipos de polímeros analizados. Ambos presentan condiciones similares en términos del análisis FTIR y de aparición de grupos funcionales debido al reprocesamiento. Sin embargo, se encontró una leve diferencia en el espectro FTIR entre estos dos polímeros debido a la presencia 
de grupos vinilo y del grupo COC. El HDPE-GI presentó mayor degradación o cambio en el espectro FTIR cuando se sometió al proceso de extrusión/inyección. EL orden de los procesos de mayor a menor efecto en la composición química del HDPE es: extrusión/inyección > inyección > extrusión a 80 rpm > extrusión a 20 rpm.

El análisis mediante FTIR del polímero después de ser reprocesado permite identificar algunos grupos tales como el grupo COC y el grupo carbonilo que no formaban parte del polímero inicial. Sin embargo, en ciertas circunstancias es difícil identificar los picos si no se cuenta con una guía metódica creada a través de la revisión bibliográfica.

\section{REFERENCIAS}

[1] N. Singh, D. Hui, R. Singh, I. Ahuja, L. Feo y F. Fraternali, "Recycling of plastic solid waste: A state of art review and future applications," Composites Part B: Engineering, vol. 115, pp. 409-422, 2017.

[2] C. Breen, P. M. Last, S. Taylor y P. Komadel, "Synergic chemical analysis - The coupling of TG with FTIR, MS and GC-MS 2. Catalytic transformation of the gases evolved during the thermal decomposition of HDPE using acid-activated clays," Thermochimica Acta, vol. 363, pp. 93-104, 2000.

[3] A. F. Rojas y T. Osswald, "Determinación de la degradación térmica de polímeros por análisis de cambio de color,” Ingeniería, vol. 21, n. ' 1, pp. 19-30, 2016.

[4] A. F. Rojas-González y L. M. Aranzazu-Ríos, "Estabilidad de procesamiento de polímeros: índice de degradación en proceso," Revista Mutis, vol. 5, n. ${ }^{\circ}$ 1, pp. 37-45, 2015. DOI: https:// doi.org/10.21789/22561498.1017

[5] A. A. Cuadri y J. E. Martín-Alfonso, "The effect of thermal and thermo-oxidative degradation conditions on rheological, chemical and thermal properties of HDPE," Polymer Degradation and Stability, vol. 141, pp. 11-18, 2017.

[6] M. J. Abad, A. Ares, L. Barral, J. Cano, F. J. Díez, S. García-Garabal, J. López y C. Ramírez, "Effects of a mixture of stabilizers on the structure and mechanical properties of polyethylene during reprocessing," Journal of Applied Polymer Science, vol. 92, n. . 6, pp. 3910-3916, 2004.

[7] S. Apone, R. Bongiovanni, M. Braglia, D. Scalia y A. Priola, "Effects of thermomechanical treatments on HDPE used for TLC ducts," Polymer testing, vol. 22, n. ${ }^{\circ}$ 3, pp. 275-280, 2003.

[8] N. Benoit, R. González-Núñez y D. Rodriguez, "High Density Polyethylene Degradation Followed by Closed- loop Recycling," Progress in Rubber, Plastics and Recycling Technology, vol. 33, n. ${ }^{\circ}$ 1, pp. 17-37, 2017.

[9] H. Hifumi, A. V. Ewing y S. G. Kazarian, "ATR-FTIR spectroscopic imaging to study the drying and dissolution of pharmaceutical polymer-based films," International Journal of Pharmaceutics, vol. 515, n. ${ }^{\text {1 }}$ 1, pp. 57-68, 2016. 
[10] T. Corrales, F. Catalina, C. Peinado, N. S. Allen y E. Fontan, "Photooxidative and thermal degradation of polyethylenes: Interrelationship by chemiluminescence, thermal gravimetric analysis and FTIR data," Journal of Photochemistry and Photobiology A: Chemistry, vol. 147, n. ${ }^{\circ} 3$, pp. 213-224, 2002.

[11] L. C. Mendes, E. S. Rufino, F. O. De Paula y A. C. Torres, "Mechanical, thermal and microstructure evaluation of HDPE after weathering in Rio de Janeiro City," Polymer Degradation and Stability, vol. 79, n. ${ }^{\circ}$ 3, pp. 371-383, 2003. DOI: https://doi.org/10.1016/S0141-3910(02)00337-3

[12] V. Parthasarathi, B. Sundaresan, V. Dhanalakshmi y R. Anbarasan, "Functionalization of HDPE with aminoester and hydroxyester by thermolysis method-An FTIR-RI approach," Thermochimica Acta, vol. 510, n. ${ }^{\circ}$ 1, pp. 61-67, 2010.

[13] C. A. Wilkie, "TGA/FTIR: an extremely useful technique for studying polymer degradation," Polymer Degradation and Stability, vol. 66, pp. 301-306, 1999.

[14] D. S. Achilias, C. Roupakias, P. Megalokonomos, A. A. Lappas y V. Antonakou, "Chemical recycling of plastic wastes made from polyethylene (LDPE and HDPE) and polypropylene (PP)," Journal of Hazardous Materials, vol. 149, n. ${ }^{\circ}$ 3, pp. 536-542, 2007.

[15] J. V. Gulmine, P. R. Janissek, H. M. Heise y L. Akcelrud, "Polyethylene characterization by FTIR,” Polymer Testing, vol. 21, n. ${ }^{\circ}$ 5, pp. 557-563, 2002.

[16] B. C. Smith, Fundamentals of Fourier transform infrared spectroscopy, London: CRC Press, 2011.

[17] Y. Kann, M. Shurgalin y R. K. Krishnaswamy, "FTIR spectroscopy for analysis of crystallinity of poly(3-hydroxybutyrate-co-4 -hydroxybutyrate) polymers and its utilization in evaluation of aging, orientation and composition," Polymer Testing, vol. 40, pp. 218-224, 2014.

[18] M. K. Loultcheva, M. Proietto, N. Jilov y F. P. La Mantia, "Recycling of high density polyethylene containers," Polymer degradation and stability, vol. 57, n. ${ }^{\circ}$ 1, pp. 77-81, 1997.

[19] P. Oblak, J. González-Gutiérrez, B. Zupančič, A. Aulova y I. Emri, "Processability and mechanical properties of extensively recycled high density polyethylene," Polymer Degradation and stability, vol. 114, pp. 133-145, 2015.

[20] I. Kriston, E. Földes, P. Staniek y B. Pukánszky, "Dominating reactions in the degradation of HDPE during long term ageing in water," Polymer Degradation and Stability, vol. 93, n. ${ }^{\circ}$, pp. 1715-1722, 2008.

[21] A. Kumar, T. Venkatappa-Rao, S. Ray-Chowdhury, y S. V. Ramana-Reddy, "Compatibility confirmation and refinement of thermal and mechanical properties of poly (lactic acid)/poly (ethylene-co-glycidyl methacrylate) blend reinforced by hexagonal boron nitride," React. Funct. Polyme., vol. 117, pp. 1-9, 2017. DOI: https://doi.org/10.1016/j.reactfunctpolym.2017.05.005

[22] A. S. F. Santos, J. A. M. Agnelli, D. W. Trevisan y S. Manrich, "Degradation and stabilization of polyolefins from municipal plastic waste during multiple extrusions under different reprocessing conditions," Polymer Degradation and Stability, vol. 77, n. 3 3, pp. 441-447, 2002.

[23] J. R. Riba, J. Cailloux, R. Cantero, R. Canals y M. L. Maspoch, "Multivariable methods applied to FTIR: A powerful technique to highlight architectural changes in poly(lactic acid)," Polym. Test., vol. 65, pp. 264-269, 2018. DOI: https://doi.org/10.1016/j.polymertesting.2017.12.003 